Chapter 9

\title{
Technological Advances in Universal Neonatal Hearing Screening (UNHS)
}

\author{
Stavros Hatzopoulos, Henryk Skarzynski and Piotr H Skarzynski \\ Additional information is available at the end of the chapter
}

http://dx.doi.org/10.5772/61178

\begin{abstract}
Within the last decade, numerous new challenges have appeared in the UNHS arena, such as (i) the need to validate the automated OAE/ABR screeners; (ii) the need to qualify the responses from the automated devices; (iii) the need to obtain additional information (i.e., hearing threshold) for the subject under assessment, in a short period of time; (iv) and the need to integrate numerous measurements in a single portable automated device. To respond to these clinical demands, several new methodologies have been introduced to the UNHS clinical practice. In this context, the aim of this chapter is to provide information on these new technological trends.
\end{abstract}

Keywords: Automated otoacoustic emissions (AOAE), automated auditory brainstem response (AABR), wideband reflectance, middle ear power analysis, neonatal hearing screening, auditory state steady response, hearing threshold

\section{Introduction}

Otoacoustic emissions (OAEs) or cochlear echoes is a term coined by David Kemp in 1978 [1], describing the transient responses from the inner ear, upon its stimulation by an acoustic click stimulus. During the last 20 years, OAE protocols have been used in many areas of audiology and hearing science [2]. The most significant contribution of OAEs is in the area of universal neonatal hearing screening (UNHS).

While the main objective of neonatal hearing screening (NHS) is the identification of infants with a hearing deficit ( $\geq 30 \mathrm{~dB} H \mathrm{HL}$ ), the objectives of a UNHS program have a broader vision. Two important phases are considered: (i) the identification of infants with mild and moderate hearing deficits and (ii) an intervention in terms of hearing improvement (hearing aids and 
cochlear implants) and neural rehabilitation, aiming at the restoration of hearing and the normalization of the quality of life of the young patient.

Within the last decade, numerous new challenges have appeared in the UNHS arena, such as (i) the need to validate the automated OAE/ABR screeners; (ii) the need to qualify the responses from the automated devices; (iii) the need to obtain additional information (i.e., hearing threshold) for the subject under assessment, in a short period of time; and (iv) the need to integrate numerous measurements in a single portable automated device. To respond to these clinical demands, several new methodologies have been introduced to the UNHS clinical practice. In this context, the aim of this chapter is to provide information on these new technological trends.

\section{Automated auditory brainstem responses}

In the early 2002, the first fourth-generation OAE devices appeared in the market and offered the possibility to integrate information from different testing protocols such as automated OAE $(\mathrm{AOAE})$ and automated $\mathrm{ABR}(\mathrm{AABR})$ responses. The combined screening protocols (AOAE + $\mathrm{AABR}$ ) targeted the identification of auditory neuropathy, most prevalent in the neonatal intensive care (NICU) environment.

With the introduction of the AABR protocols in the NHS programs, several issues became evident, and among those questions related to screening times and screening costs. The latter is outside the objectives of this paper and will not be addressed. A previous study of our group, in the context of the regional NHS project CHEAP in Emilia-Romagna, Italy [3], provided evidence suggesting that in terms of time-requirements, portable ABR (Audioscreener, Viasys; Accuscreen, GN-Otometrics; Algo 3i, Natus) and OAE devices were converging to the same time values. Data from the above study suggested that (i) the average time for AOAE responses is less than $10 \mathrm{~s}$ in a cooperative subject and less that $120 \mathrm{~s}(2 \mathrm{~min})$ in non-cooperative subjects and (ii) the average AABR test times were less than $120 \mathrm{~s}$, while longer times (600 s per ear) were required for uncooperative subjects. The placement of the AABR electrodes might be a complicated process, especially when highly skin impedances (caused by excessive lipid layers) are encountered. In these cases, the AABR algorithms tend to oversample in order to derive a coherent signal, and as a result, the testing times are significantly prolonged.

A combined two-stage approach (i.e., AOAE + AABR) eliminates the risk of not identifying infants with auditory neuropathy and assures that the screening sensitivity is high. Contrary to this hypothesis, data from a large-scale American study by White et al. [4] suggest that this is not always the case. From 86,634 screened infants, using a two-stage OAE/A-ABR protocol, $23 \%$ would have passed the AABR.

Another interesting development in the ABR/AABR area is in the area of the evoking stimulus. Traditionally, ABR and AABR protocols use click stimuli to synchronize as many neural fibers as possible and to obtain an ABR response of large amplitude with less sweeps. Recently, chirp stimuli have been used to optimize the ABR/AABR responses. According to Kristensen and 
Elberling [5], “Upward chirps are often designed to compensate for the cochlear traveling wave delay which is regarded as independent of stimulation level. A chirp based on a traveling wave model is therefore referred to as a level-independent chirp. Another compensation strategy, for instance based on frequency-specific auditory brainstem response (ABR) latencies, results in a chirp that changes with stimulation level and is therefore referred to as a level-dependent chirp. One such strategy, the direct approach, results in a chirp family that is called the levelspecific chirp." Data from studies using level-dependent chirps [6-11] are very encouraging, reporting ABRs recorded in less time and with higher amplitude values. The latter is very important for the statistical algorithms of the AABR devices, meaning that higher statistical accuracy can be obtained in the chirp-evoked AABRs.

\section{Middle ear reflectance and Middle Ear Power Analysis (MEPA)}

Data from studies that have evaluated the performance of NHS programs in the well-baby clinic or in the NICU $[4,12,13]$ have reported that the majority of "screening refers" are due to transmission impeding factors such as the amniotic fluid or any substance blocking the propagation of the acoustic stimulus. Usually, these conditions are transient (i.e., they last 24 $30 \mathrm{~h}$ ), and infants can pass the OAE test when the fluid is absorbed or when the auditory meatus is clean.

Using a middle ear power analysis (MEPA) testing procedure, it is possible to determine whether the middle ear conducts properly acoustic stimuli, and in this context, the OAE screening results can be interpreted more clearly. Data from the literature [14, 15] have shown that one of the MEPA metrics, the middle ear reflectance, is more sensitive to the distortion product OAE (DPOAE) status than the $1-\mathrm{kHz}$ tympanometry values. Power reflectance is a measure of middle ear inefficiency. It is the ratio or percentage of power reflected from the eardrum to the incident power as a function of frequency. Acoustic power measurements objectively quantify middle ear function or malfunction.

Currently, there is only one manufacturer (Mimosa Acoustics) offering reflectance measurements. The company offers two devices capable of MEPA, DPOAE, and general OAE measurements: the Otostat (handheld) and the HearID (research oriented) model. These devices (depicted in Figure 1) can measure wideband power reflectance up to $6 \mathrm{kHz}$ and most importantly without the need for a pressurized ear canal.

To interpret the clinical usefulness of the MEPA approach, Hunter et al. [15] constructed normative regions for newborns, relating middle ear reflectance values evoked by chirp stimuli and DPOAE amplitudes at 1.0, 1.5, 2.0, 3.0, 4.0, and $6.0 \mathrm{kHz}$. The three regions were described as follows:

1. A retest area (where the values of reflectance are high)

2. An ambiguous area (where the values of reflectance are moderate)

3. A pass area (where the values of reflectance are low) 

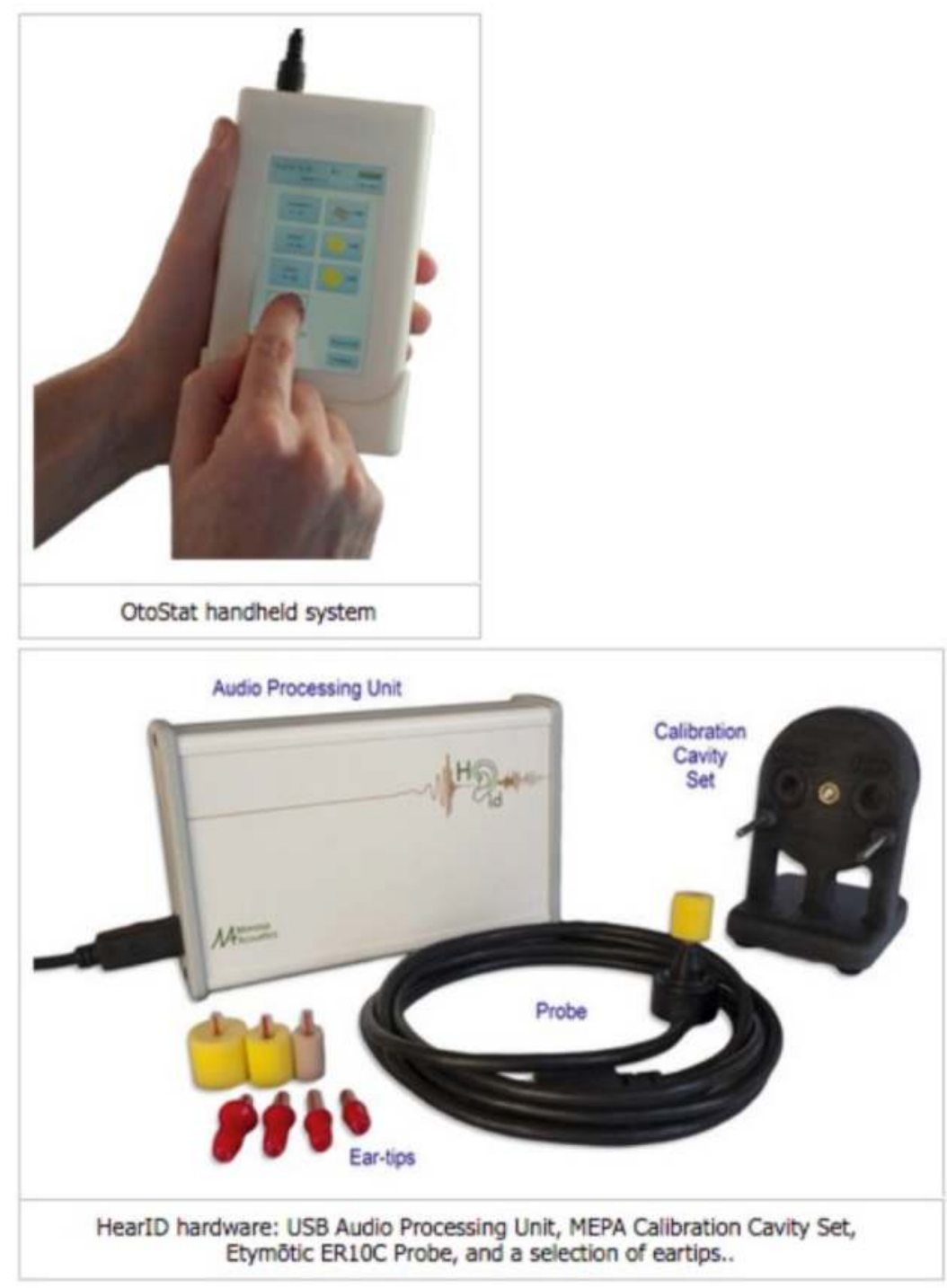

Figure 1. The Mimosa Acoustic devices capable of recording wideband reflectance and OAEs. Data from the Mimosa Acoustics website (http://www.mimosaacoustics.com).

These areas are depicted in Figure 2. In terms of interpretation, If the MEPA reflectance values fall above the "pass" area, especially around $2 \mathrm{kHz}$, outer or middle ear problems may be the cause, and a rescreening session after a few hours or a day is recommended prior to diagnostic referral. If the outcome is still a "refer" then clinical assessment is necessary. If the MEPA reflectance values fall within the "pass" area, especially around $2 \mathrm{kHz}$, the middle ear is more 
likely to be normal and associated with a DPOAE pass result. If the DPOAE result is ambiguous or a "refer", then middle ear issues are not suspected as a hearing deficit cause and further clinical assessment is necessary. Table 1 summarizes all these outcomes.

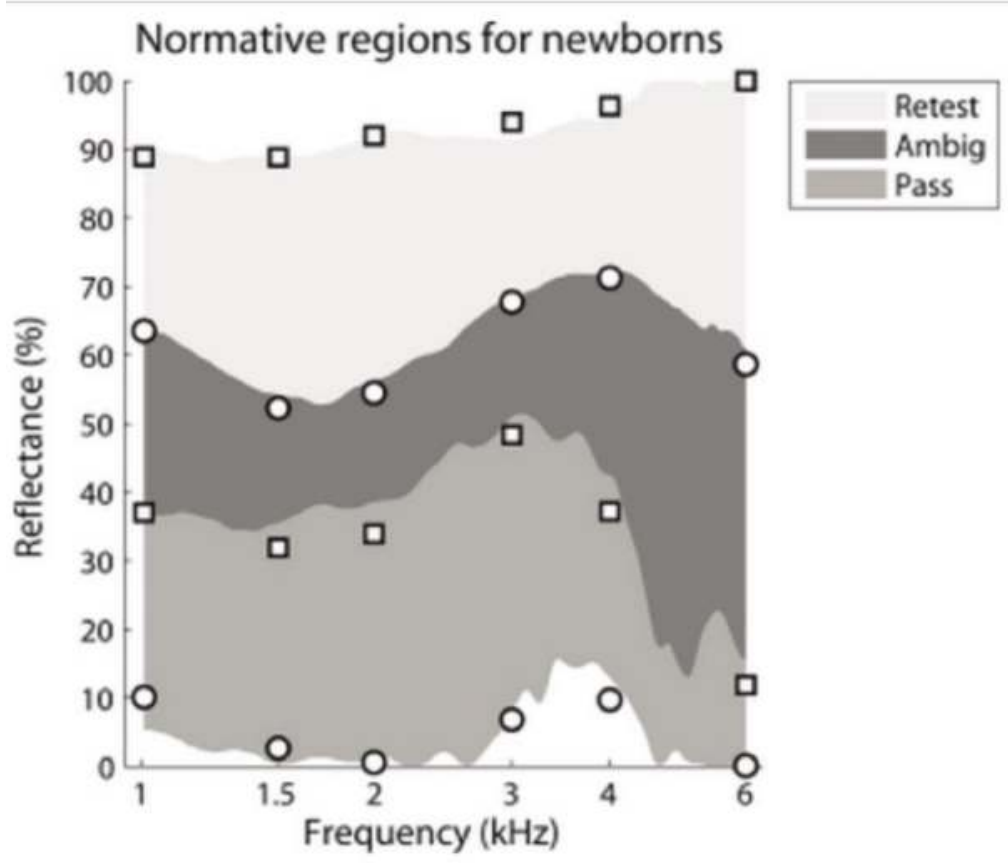

Figure 2. Pass, ambiguous, and retest regions for wideband reflectance using chirp (solid regions) and sine (symbols) stimuli. Results above this region, especially at $2 \mathrm{kHz}$, are associated with false-positive DPOAE refer results. Data from the Mimosa Acoustics website and from Hunter et al. (2010).

\begin{tabular}{|c|c|c|}
\hline Overall DPOAE result & Reflectance at $2.0 \mathrm{kHz}$ & Interpretation \\
\hline Pass & In the pass area & Pass-normal result \\
\hline Pass & $\begin{array}{l}\text { Above the pass area (i.e., in the ambiguous or } \\
\text { retest area) }\end{array}$ & $\begin{array}{l}\text { Pass - may have middle ear issues, } \\
\text { cochlear response normal }\end{array}$ \\
\hline Refer & In the pass area & $\begin{array}{l}\text { Refer - consistent with SNHL, requires } \\
\text { follow-up }\end{array}$ \\
\hline Refer & $\begin{array}{l}\text { Above the pass area (i.e., in the ambiguous or } \\
\text { retest areas) }\end{array}$ & $\begin{array}{l}\text { Rescreening is suggested; repeat MEPA to } \\
\text { determine status of the middle ear }\end{array}$ \\
\hline
\end{tabular}

Table 1. How to interpet distortion product OAEs and reflectance results in newborns (from http:// www.mimosaacoustics.com). 


\section{Auditory Steady-State Responses (ASSR)}

$\mathrm{OAE}$ and $\mathrm{ABR}$ testing procedures are evoked by electrical transient stimuli (clicks, filtered clicks, etc.), and as a result, the responses are correlated with a few audiometric frequencies, which correspond to the maximum spectral content of the stimulus (around $2.0 \mathrm{kHz}$ ). Considering this clinical setup, there are other protocols that could be candidates for a hearing assessment of neonates, children, and adults. Among those is the electrocochleography (EcoG), the middle latency (ML) responses, and the most recently reported steady-state responses (SSR). The first two approaches can be excluded because they require long times either for the position of an intratympanic electrode or for sampling purposes. The last protocol has shown a good potential for hearing screening since with an adequate manipulation of the stimulus modulation frequency, one can record responses or from the auditory cortex (low modulation frequencies around $40 \mathrm{~Hz}$ ) or from the brainstem (frequencies around 50-120 Hz) [16-18]. The basic SSR protocol has evolved into an automated procedure (ASSR) where multiple stimulus frequencies are used and regression models predict hearing levels at the tested stimuli. The ASSR protocols have been greatly optimized for lower frequency stimuli such as $500 \mathrm{~Hz}$ [19].

In 2002, Conne-Wesson et al. [16] suggested that it could be possible to use an SSR protocol in a Neonatal Hearing Program, and since the SSR responses were generated by the brainstem for modulation frequencies $>40 \mathrm{~Hz}$, the ASSR could substitute the AABR [20-22]. In the referenced studies, a good agreement has been reported between the ASSR and the AABR responses at $2.0 \mathrm{kHz}$ and various significant differences at $0.5,1.0$, and $4.0 \mathrm{kHz}$. The available data suggest that the AASR protocols should be developed further to become more independent of various clinical factors (related to the tested subject and to the stimuli used) and should be applied on a large population of subjects so that the results can be easily used clinically.

The important factors affecting the AABR responses (i.e., the ambient noise and the skinelectrode impedance) interfere also with the ASSR recordings. In 2010, Vivosonic presented a new family of devices (called amplitrodes) using a novel approach. Each scalp electrode was connected to a small preamplifier within the electrode assembly. Amplifying the signal in situ has many advantages, such as the suppression of the ambient noise and the elevation of the signal-to-noise ratio $(\mathrm{S} / \mathrm{N})$. This approach results in clean AABR and ASSR traces. One of the issues reported since its release, is that the new electrodes require very often a change of the electrode batteries.

In the context of a neonatal screening, an ASSR screening protocol can focus on discrete frequency points (i.e., 1.0 and $2.0 \mathrm{kHz}$ or 2.0 and $4.0 \mathrm{kHz}$ ), which show relative immunity to ambient noise, as shown in the neonatal data in Figures 3A and 3B. One of the problems of the early ASSR devices (Audera by Viasys; Master by Natus) was that the mean hearing threshold estimates were characterized by large variance. Recent data in the literature and specifically from the Audix equipment developers (Neuronic) report significant advances both in terms of software and hardware and a superior performance of a multiple SSR protocol to the conventional ABR $[23,24]$. 
A

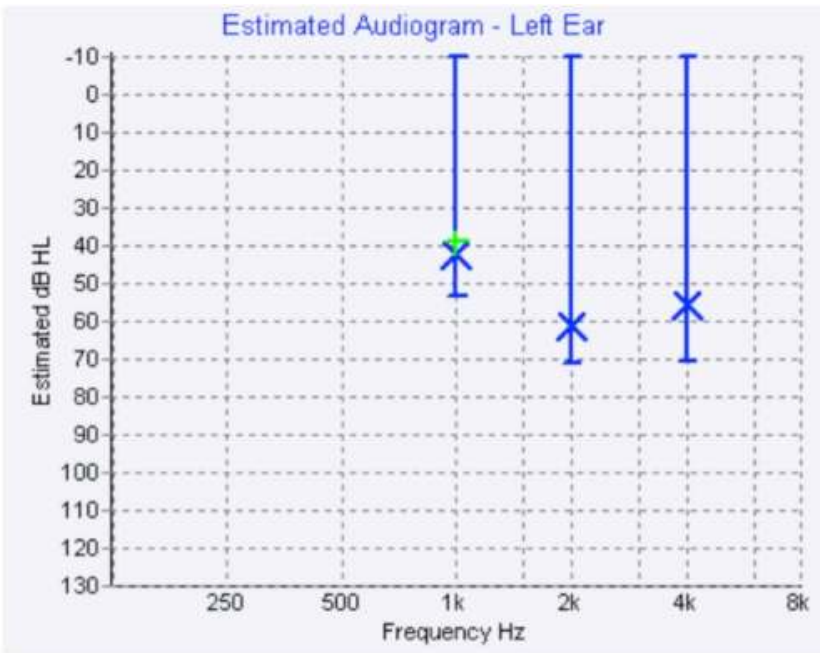

B

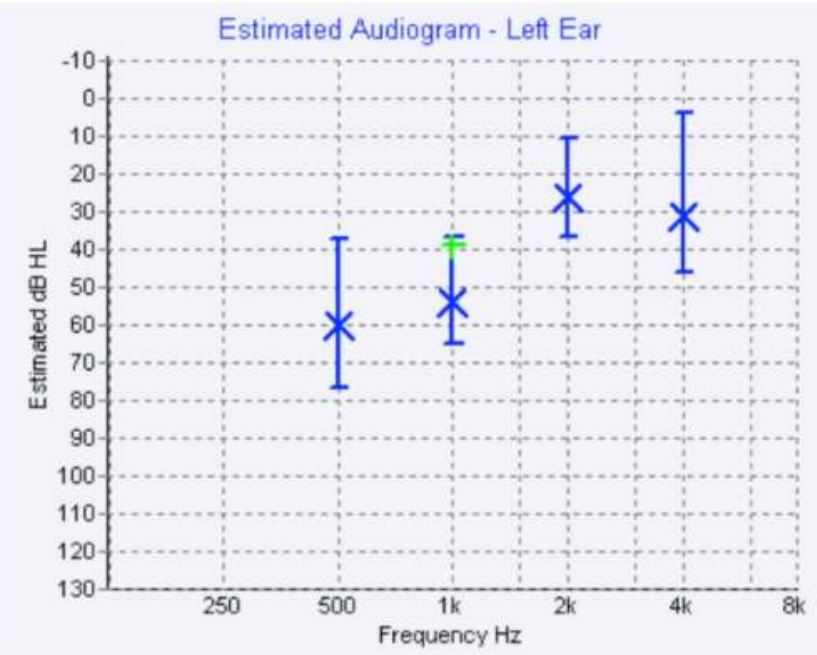

Figure 3. A) ASSR responses from a non-cooperative infant, using the AUDERA ASSR device (VIASYS). Responses at $500 \mathrm{~Hz}$ were not available due to noise caused by myogenic artifacts. The ASSR recording time was 14 min longer that the AABR test, resulting as $22 \mathrm{~min}$. The large size of the error bars, at 2.0 and $4 \mathrm{kHz}$, show threshold means at 60 and $55 \mathrm{~dB}$ HL, but the variability of the measurements makes the threshold prediction difficult to be considered. (B) ASSR response from another well-baby infant, using the same ASSR device. The ASSR recording was also significantly longer than the AABR response ( $16 \mathrm{vs} .7 \mathrm{~min}$ ). The error bars around the threshold average (indicated by an " $\mathrm{x}$ ") are small and the prediction can be considered practical. For example, at $1.0 \mathrm{kHz}$, the threshold level is shown at $55 \mathrm{~dB}$ with a $95 \%$ probability that it will be in the interval 35-65 dB HL. The latter estimates are derived from the values of the error bars. 
Recently, a study by Ciorba et al. [25] presented data on the relationship between ABR, ASSR estimates, and data from Conditioned Orientation Responses (COR), a technique widely diffused in the intervention phase of many UNHS programs. The data suggested a very good relationship between the outcomes of the ASSR and the COR techniques, with the ASSR data being closer to the ABR estimates. Data from large-scale studies along this direction (i.e., comparing ASSR with other protocols) could support this hypothesis and eliminate the use of $\mathrm{ABR}$ and $\mathrm{COR}$ in this intervention step.

\section{Threshold estimation via DPOAE measurements}

From the early nineties, where OAEs were accepted in the clinical practice, the relationship between hearing threshold and OAE responses received a lot of attention [26-28]. What previous research suggests is that in cases presenting sensorineural deficits (i.e., excluding conductive and retrocochlear causes), there is a good agreement between the OAE respond levels and the hearing threshold. In this context, distortion product OAE (DPOAE) protocols can provide additional information [26, 29-31]. Input-output (or I/O functions) DPOAE protocols provide information on the relationship between the evoking stimulus and the signal compression of the cochlear amplifier. Data supporting this hypothesis are derived from animal experiments (furosemide intoxication) [32] and clinical human studies from cases presenting sensorineural deficits [29, 33-34]. When the hearing loss is increased, the slope of the corresponding DPOAE I/O-functions decreases and reveals a loss of compression in the cochlear amplifier. Using various setups of the DPOAE I/O stimuli, one can estimate the cochlear compression, which is related to a specific threshold value [31, 35]. Janssen et al. [36] used this concept to produce a relationship between DPOAE I/O amplitude values and hearing threshold. According to their data, "The hearing threshold was found to be increasing within the early postnatal period (average age: 3 days), predominantly at the higher frequencies, and to be normalized in a follow-up measurement (after four weeks). However, the slope of the DPOAE I/O-functions obtained in the first and second measurement was unchanged revealing normal cochlear compression. Consequently, these findings were interpreted as temporary conductive hearing losses due to the presence of amniotic fluid and/or Eustachian tube dysfunction." The value of cochlear compression changes when the middle ear stimulus pathway is affected. Therefore, this procedure has the theoretical potential to discriminate middle from inner ear deficits. Data from the literature have not validated yet this hypothesis.

The research findings from Janssen et al. [36] and Gorga et al. [35] have been commercialized by Natus in the Cochlea-Scan device [37]. Hearing threshold can be extrapolated up to values relative to $50 \mathrm{~dB} \mathrm{HL}$ in the frequency range from 1.5 to $6 \mathrm{kHz}$. Figure 4 shows a typical hearing threshold profile and the corresponding Cochlea-Scan-mediated estimation of hearing threshold. At present, the Cochlea-Scan device offers a platform for a third-generation OAE testing (TEOAEs and DPOAEs), I/O DPOAE estimation with hearing threshold extrapolation.

Further analyses [38, 39] on the efficacy of the Cochlea-Scan DPOAE algorithm, relating hearing threshold data and Cochlea-Scan estimated thresholds from a group of adult sensor- 

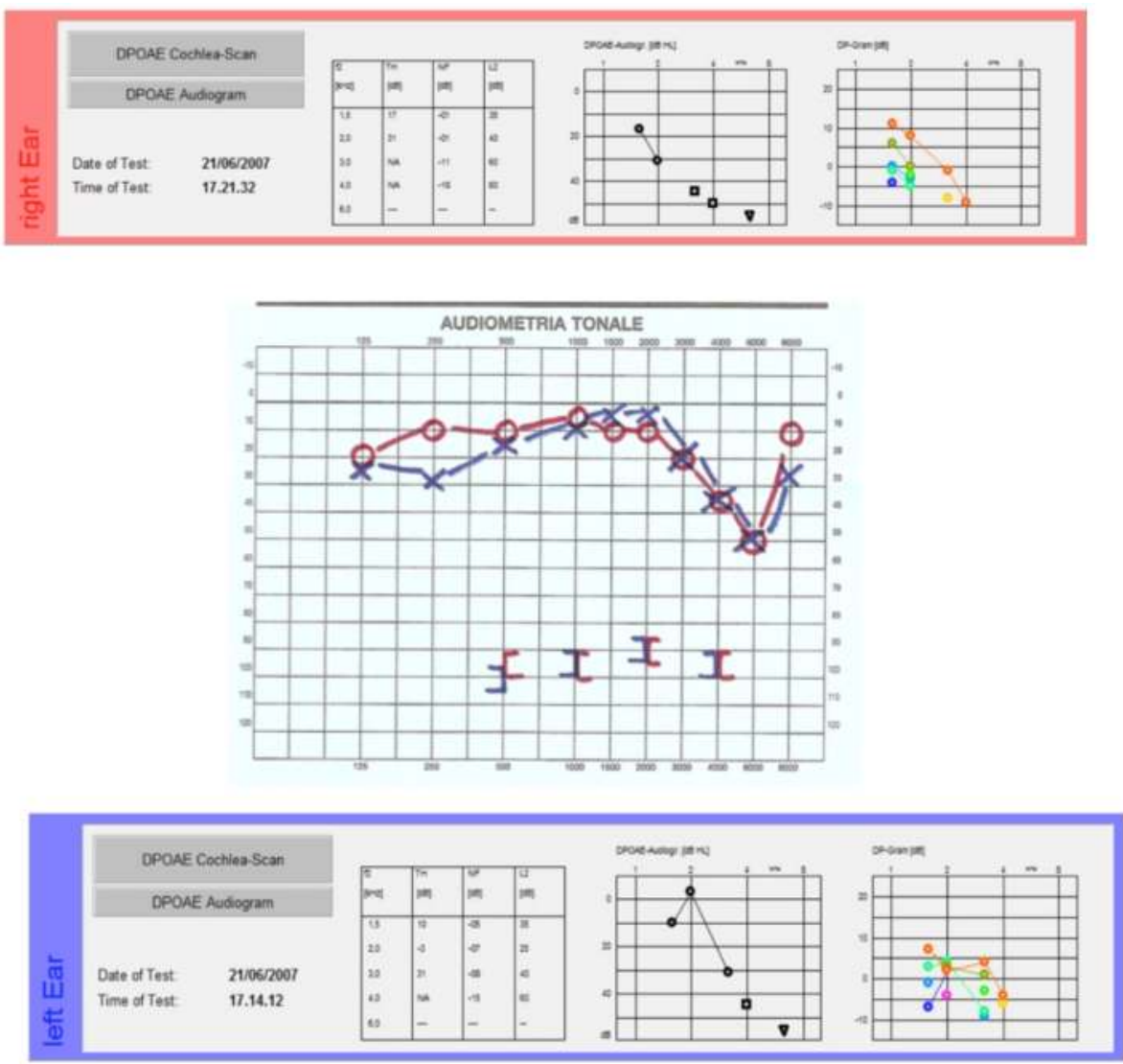

Figure 4. Cochlea-Scan data in comparison to behavioral threshold levels, from an adult subject. Top panel: CochleaScan responses and threshold estimation from the right ear; middle panel: behavioral data; bottom panel: Cochlea-Scan responses and threshold estimation from the left ear. The Cochlea-Scan panels report the estimated threshold values per frequency. The acronym "NA" means that at the specific frequency no threshold estimation was possible.

ineural cases, suggested a different scenario than the one proposed initially by Janssen et al. [36]. In the Hatzopoulos et al. [39] study, behavioral and Cochlea-Scan data were analyzed with logistic regression models in order to find the probability $(\leq 0.9)$ of a robust DPOAE response at 2.0,3.0, and $4.0 \mathrm{kHz}$. The data suggested that the maximum behavioral levels where valid DPOAEs could be detected were equal to of $32.8,21$, and $34 \mathrm{~dB}$, respectively. For normal hearing adults, the detection levels were lower. Figures 5 and 6 depict the relationship between behavioral data (at 2.0,3.0, and $4.0 \mathrm{kHz}$ ) and Cochlea-Scan estimates from the cases presenting hearing loss. For example, in Figure 5 and for $2.0 \mathrm{kHz}$, a probability of $90 \%$ Cochlea-Scan response detection corresponds to a threshold approximately of $15 \mathrm{~dB} \mathrm{HL}$. In this context, it is still possible to have a detection threshold as high as $50 \mathrm{~dB}$ HL. The corresponding probability falls below $30 \%$ and, as such, limits the usefulness of the Cochlea-Scan protocol 
HL Predicted $220=\exp \left(3.6079-.0802^{*} p 20\right) /\left(1+\exp \left(3.6079-.0802^{*} p 20\right)\right)$

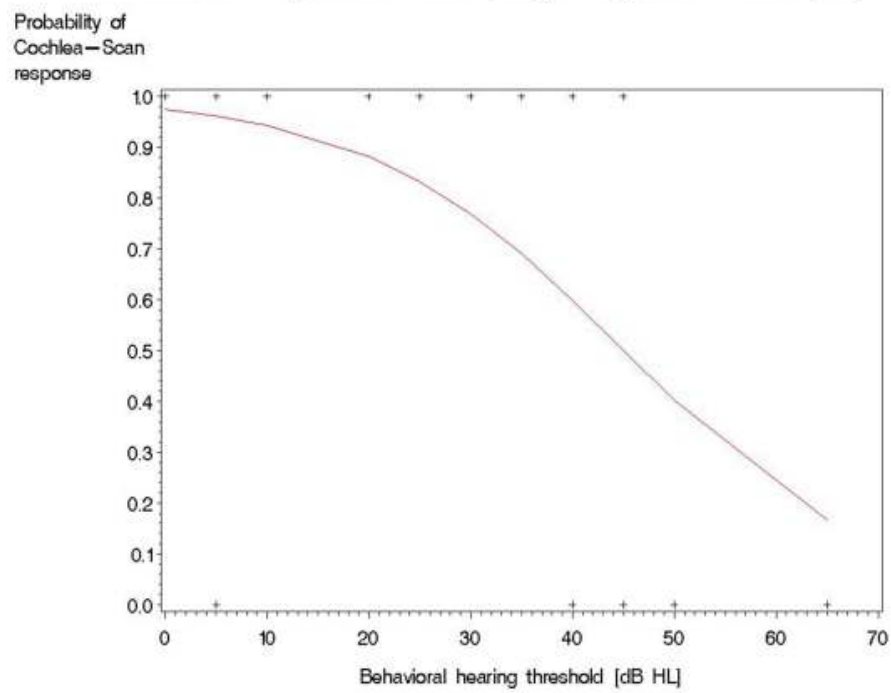

HL Predicted $\mathcal{C}^{30}=\exp \left(5.4447-.1507^{\star} p 30\right) /\left(1+\exp \left(5.4447-.1507^{\star} p 30\right)\right)$

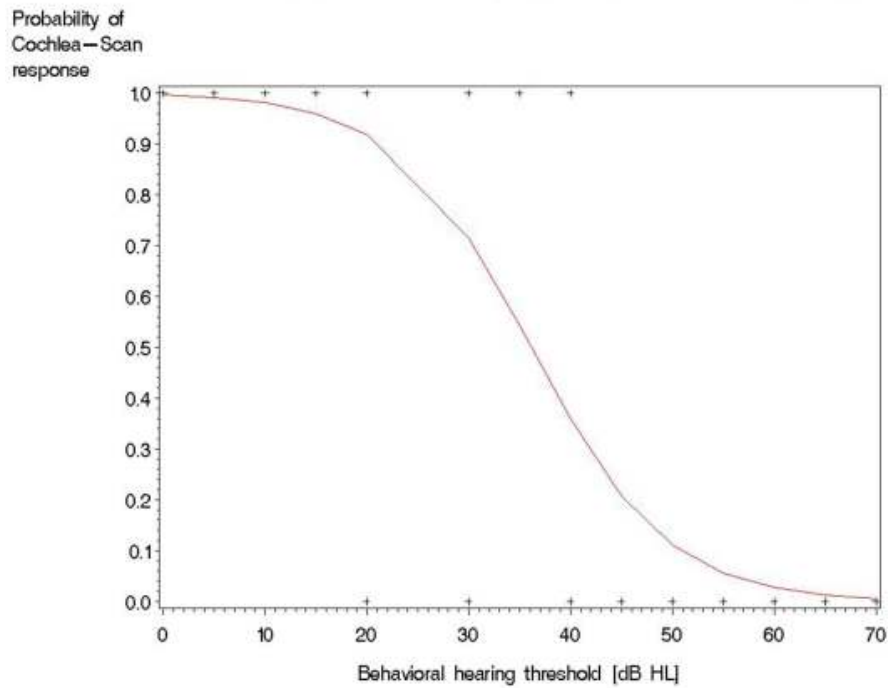

Figure 5. Logistic regression model for normal hearing threshold Cochlea-Scan data at 2.0 and $3.0 \mathrm{kHz}$. The equation relating the two variables ( $\mathrm{c}=$ Cochlea-Scan; $\mathrm{p}=$ behavioral threshold) is shown at the top of each graph. The $x$ axis shows behavioral threshold in $\mathrm{dB}$ HL and the $y$ axis the probability of a Cochlea-Scan response. For a fixed response probability of $90 \%$, the detectable threshold level is approximately 15 and $20 \mathrm{~dB} \mathrm{HL}$, for the data at 2.0 and $3.0 \mathrm{kHz}$. This implies that in order to obtain a Cochlea-Scan response for a 50-dB HL hearing threshold, the probability of finding a true response drops to $40 \%$ and $10 \%$, respectively (for 2.0 and $3.0 \mathrm{kHz}$ ). 


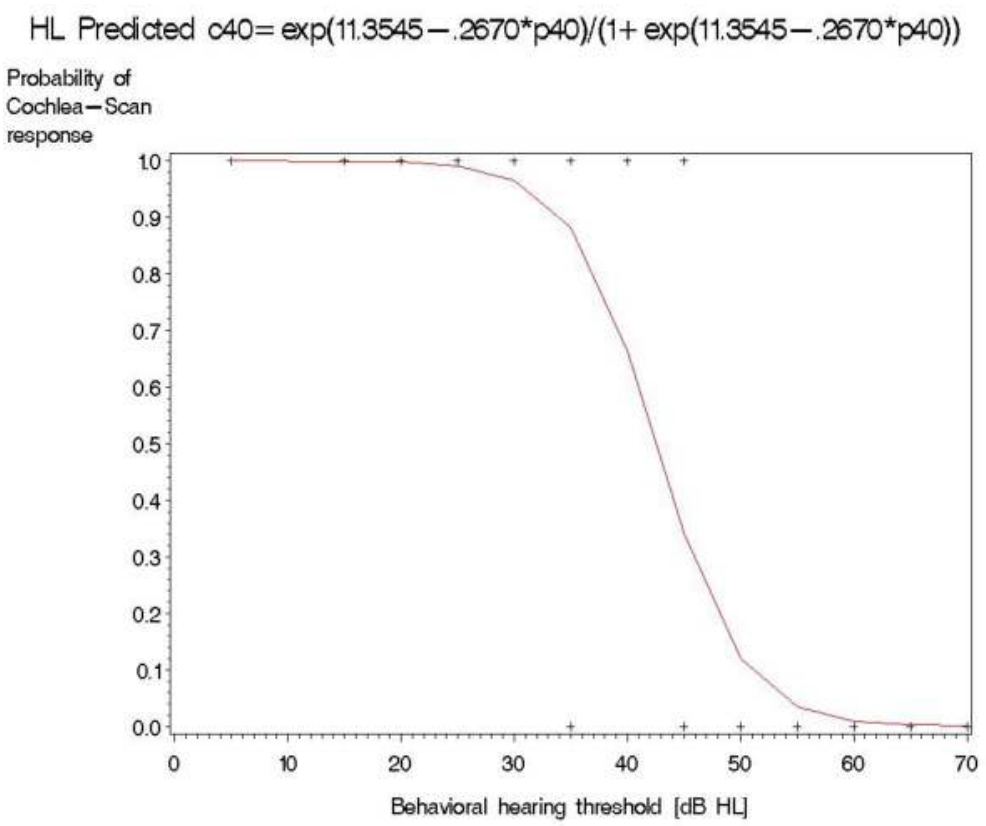

Figure 6. Logistic regression model for normal hearing threshold Cochlea-Scan data at $4.0 \mathrm{kHz}$. The equation relating the two variables $(\mathrm{c}=$ Cochlea-Scan; $\mathrm{p}=$ behavioral threshold) is shown at the top of each graph. The $x$ axis shows behavioral threshold in $\mathrm{dB}$ HL and the $y$ axis the probability of a Cochlea-Scan response. For a fixed response probability of $90 \%$, the detectable threshold level is approximately $35 \mathrm{~dB}$ HL. For a $50-\mathrm{dB}$ HL threshold, the probability of a true response drops to $15 \%$. The relationship between the behavioral and the Cochlea-Scan data at $4.0 \mathrm{kHz}$ is optimized, but the sensitivity of the method drops very quickly as we move to higher thresholds $35 \mathrm{~dB}$ HL.

The authors at this point in time could not verify if Natus has intentions of developing further this product. Cochlea-Scan threshold estimation could be greatly improved by introducing changes in the device's algorithms related to (i) the sample size, which was used to calibrate the prototype device. Sampling a larger population can minimize the variance of the average DPOAE amplitude per tested frequency (ii) by inserting correction factors in the algorithm, which extrapolates DPOAE amplitudes to hearing levels. Janssen et al. [36] have used a linear regression model to achieve this, but higher-order models (quadratic, cubic) can offer higher precision in the threshold estimation.

\section{Integration of multiple hearing assessment protocols into an automated device}

The success of the NHS screening practices challenged another area of pediatric audiology, the area of schoolchildren screening. Data from large-scale screening programs, as in Poland, suggested that in this area different protocols could be applied than in UNHS programs, with 
emphasis on pure tone behavioral responses, tympanometry, and ABR [40]. The OAEs were found the less effective tool in the battery of screening tests, suffering mainly from the ambient noise present in schools.

Recently, the fifth-generation OAE equipment appeared in the market. A number of OAE manufacturers (Natus, Path Medical solutions) proposed handheld devices capable of testing subjects with OAEs/AOAEs, AABR, and ASSR. A tympanometry assessment has not appeared so far due to complications in the probe of the device (canal pressurization issues). Mimosa Acoustics offers wide-reflectance measurements (which can substitute acoustic immittance) and OAEs but not evoked potentials.

The proposal from Path Medical Solutions (model: Sentiero-advanced) is a device capable not only of AOAE/AABR/ASSR protocols but also of protocols for speech Audiometry. The device is depicted in Figure 7. Such a device can be easily implemented in both phases (identification and intervention) of a UNHS program, and it is hoped that other manufacturers will follow this protocol-integration trend.

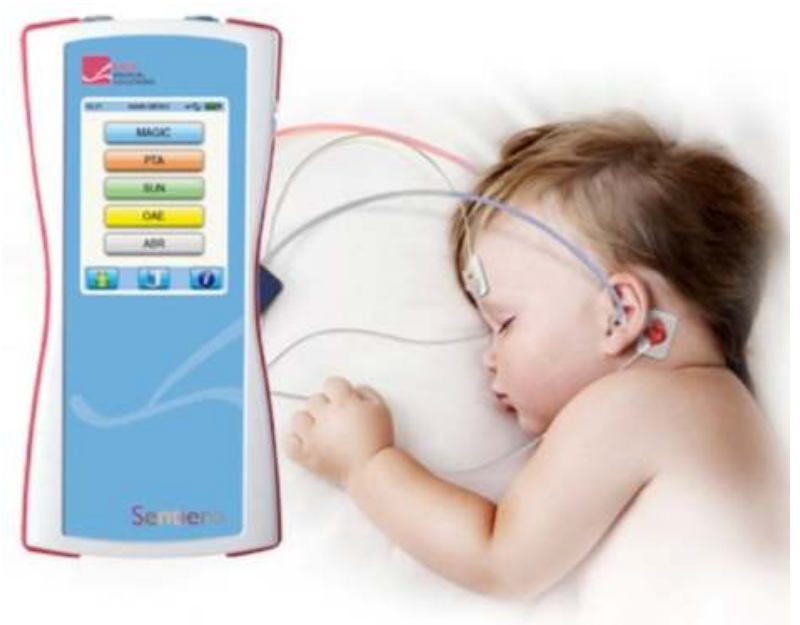

Figure 7. The Sentiero Advanced device (data from the website of Path medical solutions http://www.pathme.de).

\section{Conclusions}

During the last 10-15 years, significant advances have been made toward the integration of various protocols and technologies in UNHS strategies. The most important contribution is in the area of auditory steady-state responses, which has been shown to be well correlated with other metrics in audiology such as the AABR, ABR, OAEs, and COR. The current technological trends call for an integration of even more protocols and algorithms in a handheld device. The clinical robustness and response quality of these new entries is yet to be evaluated. 


\section{Appendix}

The reader interested in additional information than the one presented might visit the OAE Portal (http://www.otoemissions.org) and the OAE Portal Forum.

\section{Author details}

Stavros Hatzopoulos ${ }^{1}$, Henryk Skarzynski ${ }^{2}$ and Piotr H Skarzynski ${ }^{2,3,4}$

1 Department of Audiology and ENT, University of Ferrara, Italy

2 Institute of Physiology and Pathology of Hearing, Warsaw, Poland

3 Heart Failure and Cardiac Rehabilitation Department of the Medical University of Warsaw, Poland

4 Institute of Sensory Organs, Kajetany, Poland

\section{References}

[1] Kemp DT. Stimulated acoustic emissions from within the human auditory system. J Acoust Soc Am. 1978:1386-91.

[2] Robinette $\mathrm{M}$ and Glattke $\mathrm{T}$ editors. Otoacoustic Emissions: Clinical Applications. New York: Thieme; 1997.

[3] Ciorba A, Hatzopoulos S, Camurri L, Negossi L, Rossi M, Cosso D, and Martini A. Neonatal newborn hearing screening: four years of experience at the Ferrara University Hospital (cheap project). Acta Otorhinolaryngol Ital, 2007;27:10-16.

[4] White KR, Vohr BR, Meyer S, Widen JE, Johnson JL, Gravel JS, James M, Kennalley T, Maxon AB, Spivak L, Sullivan-Mahoney M, Weirather Y. A multisite study to examine the efficacy of the otoacoustic emission/automated auditory brainstem response newborn hearing screening protocol: research design and results of the study. Am J Audiol. 2005;14:186-199.

[5] Kristensen SG, Elberling C. Auditory brainstem responses to level-specific chirps in normal-hearing adults. J Am Acad Audiol 2012; 23:712-721.

[6] Ferm I, Lighfoot G, Stevens J. Comparison of ABR response amplitude, test time and estimation of hearing threshold using specific chirp and tone pip stimuli in newborns. Int J Audiol 2013;52:419-423. 
[7] Rodriguez GR, Ramos N, Lewis DR. Comparing auditory brainstem responses to toneburst and narrow band CE-chirp in young infants. Int J Pediatr Otorhinolaryngol 2013;77:1555-1560.

[8] Zirn S, Louza J, Reiman V, Wittlinger N, Hempel JM, Schuster M. Comparison between ABR with click and narrow band chirp stimuli in children. Int J Pediatr Otorhinolaryngol 2014; 78:1352-1355.

[9] Cebulla M, Lurz H, Shehata-Dieler W. Evaluation of waveform, latency and amplitude values of chirp ABR in newborns. Int J Pediatr Otorhinolaryngol 2014; 78: 762768.

[10] Rodriguez GR, Lewis DR. Establishing auditory steady-state response thresholds to narrow band CE-chirps in full term neonates. Int J Pediatr Ororhinolaryngol 2014;78:238-243.

[11] Stuart A, Cobb KM. Effect of stimulus and number of sweeps on the neonate auditory brainstem response. Ear Hear 2014;35:585-588.

[12] Aithal S, Aithal V, Kei J, Driscoll C. Conductive hearing loss and middle ear pathology in young infants referred through a newborn universal hearing screening program in Australia. J Am Acad Audiol 2012 23: 673-685.

[13] Vos B, Lagasse R, Leveque A. Main outcomes of a newborn hearing screening program in Belgium over six years. Int J Pediatr Otorhinolaryngol 2014 78:1496-1502.

[14] Sanford C, Keefe D, Liu YW, Fitzpatrick D, McCreery R, Lewis D, and Gorga M. Sound-conduction effects on distortion-product otoacoustic emission screening outcomes in newborn infants: test performance of wideband acoustic transfer functions and $1 \mathrm{kHz}$ tympanometry. Ear Hear 2009;30: 635-652.

[15] Hunter L, Feeney P, Miller JL, Jeng P, and Bohning S. Wideband reflectance in newborns. Normative regions and relationship to hearing-screening results. Ear Hear 2010;31:599-610.

[16] Cone-Wesson B, Dowell R, Tomlin D, Rance G, Ming Wu. The auditory steady state response. Comparisons with the auditory brainstem response. J Am Acad Audiol 2002; 13: 173-187.

[17] Dimitrijevic A, John S, Roon P, et al. Estimating the audiogram using multiple auditory steady state responses. J Am Acad Audiol 2002; 13: 205-224.

[18] John MS, Picton TW. Human auditory steady-state responses to amplitude-modulated tones: phase and latency measurements. Hear Res 2000; 141: 57-79.

[19] Gorga MP, Neely ST, Hoover BM, Dierking DM, Beauchaine KL, Manning C. Determining the upper limits of stimulation for auditory steady-state response measurements. Ear Hear. 2004;25:302-307. 
[20] Stueve MP, O'Rourke C. Estimation of hearing loss in children: comparison of auditory steady-state response, auditory brainstem response, and behavioral test methods. Am J Audiol. 2003;12:125-136.

[21] Luts H, Desloovere C, Kumar A, Vandermeersch E, Wouters J. Objective assessment of frequency-specific hearing thresholds in babies. Int J Pediatr Otorhinolaryngol. 2004 68(7):915-926.

[22] Swanepoel D, Hugo R, Roode R. Auditory steady-state responses for children with severe to profound hearing loss. Arch Otolaryngol Head Neck Surg. 2004;130:531535.

[23] MIjares E, Perez Abalo MC, Herrera D, Lage A, Vega M. Comparing statistics for objective detection of transient and steady-state evoked responses in newborns. Int J Audiol 2013;52:44-49.

[24] Perez Abalo MC, Rogriguez E, Sanchez M, Santos E, Torres Fortuny A. New system for neonatal hearing screening based on auditory steady state responses. J Med Eng Technol 2013;37:368-374.

[25] Ciorba A, Hatzopoulos S, Petruccelli J, Mazzoli M, Pastore A, Kochanek K, Skarzynski P, Wlodarczyk A and Skarzynski H.Identifying congenital hearing impairment: preliminary results from a comparative study using objective and subjective audiometric protocols. Acta Otorhinolaryngol Ital 2013;33:29-35.

[26] Whitehead ML, McCoy MJ, Lonsbury-Martin BL, Martin GK. Dependence of distortion-product otoacoustic emissions in primary tone level in normal and impaired ears. I. Effects of decreasing L2 below L1. J Acoust Soc Am 1995a 97: 2346-2358.

[27] Whitehead ML, Stagner BB, Lonsbury-Martin BL, Martin GK. Effects of ear-canal standing waves on measurements of distortion-product otoacoustic emissions. J Acoust Soc Am 1995b 98: 3202-3214

[28] Shera CA, Guinan JJ Jr. Evoked otoacoustic emissions arise by two fundamentally different mechanisms: a taxonomy for mammalian OAEs. J Acoust Soc Am. 1999;105:782-798.

[29] Janssen T, Kummer P, Arnold W. Growth behavior of the 2f1-f2 distortion product otoacoustic emission in tinnitus. J Acoust Soc Am 1998;103 (6):3418-3430.

[30] Dorn PA, Konrad-Martin D, Neely ST, Keefe DH, Cyr E, Gorga MP. Distortion product otoacoustic emission input/output functions in normal-hearing and hearing-impaired human ears. J Acoust Soc Am. 2001;110:3119-3131.

[31] Gorga MP, Neely ST, Dierking DM, Dorn PA, Hoover BM, Fitzpatrick DF. Distortion product otoacoustic emission suppression tuning curves in normal-hearing and hearing-impaired human ears. J Acoust Soc Am. 2003b;114(1):263-278. 
[32] Mills DM, Rubel ED. Development of the cochlear amplifier. J Acoust Soc Am 1996; 100: $428-441$.

[33] Kummer P, Janssen T, Arnold W. The level and growth behavior of the 2f1- $\mathrm{f} 2$ distortion product otoacoustic emission and its relationship to auditory sensitivity in normal hearing and cochlear hearing loss. J Acoust Soc Am 1998;103 (6):3431-3444.

[34] Boege P, Janssen T. Pure-tone threshold estimation from extrapolated distortion product otoacoustic emission I/O-functions in normal and cochlear hearing loss ears. J Acoust Soc Am 2002 111: 1810-1818.

[35] Gorga MP, Neely ST, Dorn PA, Hoover BM. Further efforts to predict pure-tone thresholds from distortion product otoacoustic emission input/output functions. J Acoust Soc Am 2003a;113:3275-3284.

[36] Janssen T, Klein A, Gehr D. Automatische Hörschwellenbestimung bei Neugeborenen mit extrapolierten DPOAE-Wachstumsfunktionen. Eine neue HörscreeningMethode. HNO $200316: 125-128$

[37] Oswald JA, Janssen T Weighted DPOAE I/O-functions: a tool for automatically assessing hearing loss in clinical application. Z Med Physik 2003; 13: 93-98.

[38] Hatzopoulos S, Petruccelli J, Ciorba A and Martini A. Optimizing otoacoustic emission protocols for a UNHS program. Audiol Neurotol 2009;14(1):7-16.

[39] Hatzopoulos S, Ciorba A, Petruccelli J, Grasso D, Sliwa L, Skarzynski H and Martini A. Estimation of the Hearing Threshold Using behavioral Audiometry, CochleaScan and ASSR protocols Int J Audiol 2009;48:625-631.

[40] Sliwa L, Hatzopoulos S, Kochanek K, Piłka A, SenderskiA and Henryk Skarzynski. A comparison of audiometric and objective methods in hearing screening of school children. A preliminary study. Int J Pediatr Otorhinolaryngol 2011;75: 483-488. 